immune-regulating response when exposed to an inflammatory environment through the secretion of soluble mediators and cell-cell contact mechanisms. Several molecules are implicated in ADSC-mediated immunomodulation such as PGE2, IDO, IL-6, TGF- $\beta$ and TSG6. However, while ADSC have shown remarkable results in vitro and in animal models, their efficacy in clinical applications has yet to be fully elucidated.

Objectives Considering the diversity of the local inflammatory microenvironment in the joints of RA patients and to further explore the behaviour of ADSC within their microenvironment, we aimed to study the effect of synovial fluid (SF) collected from patients with RA on ADSC proliferation and immune-modulating properties.

Methods ADSC isolated from 6 healthy subjects were cultured for 24 hours in the presence or absence of RASF (diluted to $5 \%$ ) obtained from 10 different RA patients or a control SF obtained from a patient undergoing non-inflammatory rheumatism related surgery. ADSC proliferation was assessed by an MTT colorimetric assay. After SF treatment, ADSC were harvested for RNA extraction and subsequent RT-qPCR to quantify the gene expression of COX2, IL6, IDO, TSG6, TGF- $\beta$. To evaluate the inflammatory status of SF, pro-inflammatory cytokines present in SF were first identified using an antibody-based membrane array and then quantified by ELISA or CBA.

Results RASF differentially affected the proliferation of ADSC with 2 out of 10 inducing ADSC proliferation whilst the remaining inhibited proliferation. Gene expression of COX2, IDO, IL-6, TSG6 and TGF- $\beta$ was simultaneously induced in only 4 of the 10 RASF included in this study. We thus investigated if these four RASF had any particular inflammatory signature that could explain their effect on ADSC. Higher concentrations of one or more of the following markers IL-6, TNF $\alpha$, MCP1, ICAM1, IL1ra were detected in these SF compared to others, which correlates with a more potent proinflammatory environment. Conversely, RASF that had no effect on ADSC gene expression presented a less inflammatory profile. Recombinant cytokines and neutralisation assays are underway to determine which specific molecules mediated the SF effect on ADSC.

Conclusions Proper licensing of ADSC to an anti-inflammatory phenotype is usually done in vitro by stimulation with high concentrations of pro-inflammatory cytokines which do not necessarily reflect the concentrations present in the synovial fluid. This study sheds further light on how ADSC respond to local inflammatory stimuli in order to better direct their immunomodulatory ability and efficacy in clinical therapy.

Acknowledgements $* \mathrm{~N}$. Alaaeddine and J.-L. Davignon are joint senior authors

Disclosure of interest None declared

\section{P122 TREATMENT WITH RITUXIMAB TO DEMAND IN RHEUMATOID ARTHRITIS. EFFECTIVENESS ANALYSIS}

${ }^{1} \mathrm{U}$ Julia*, ${ }^{2} \mathrm{MJ}$ Fobelo, ${ }^{2} \mathrm{M}$ Guzman. ${ }^{1}$ Rheumatology Unit; ${ }^{2}$ Pharmacy Unit, Valme University Hospital, Seville, Spain

\subsection{6/annrheumdis-2018-EWRR2018.137}

Introduction Rituximab (RTX) is a chimeric anti-CD20 monoclonal antibody that has been shown to be effective in the treatment of rheumatoid arthritis (RA). At present we have different treatment regimens in terms of the time of administration, every 6 months or on demand and with respect to the dose used: cycles of $1000 \mathrm{mg}$ x 2 or $500 \mathrm{mg} \times$ 2.

Objectives Our objetive was to analyse the effectiveness ondemand tratment regimen of RTX in RA

Methods Retrospective observational study on RA with RTX treatment between January 2006 and February 2015 was carried out. Administration was performed if DAS28 >3.2. The median time of retreatment and the number of patients portrayed at 8,12, 16, 20 and 24 months were calculated

Results 57 patients were included, mean age 55 years, $70 \%$ women; 93\% FR+, 31.6\% ACPA + and 63.2\% with erosive disease. The median time of evolution of RA at the start of RTX 8.1 years. All patients had high activity at the start of RTX [median DAS28 $=5.6 ; \mathrm{HAQ}=1.75$ ]. Prior to RTX, all patients were treated with another biological $(24.5 \%$ with two or more). $56 \%$ of patients were receiving DMARDs.

The median time of retreat was 7 months.

The number of patients portrayed was: at 8 months $55.8 \%$, at 12 months $69.4 \%$, at 16 months $80 \%$, at 20 months $81.3 \%$ and at 24 months at $88.6 \%$. Those not evaluated in each quarter are those who are discontinued RTX before two years of follow-up (four due to inefficacy and one due to adverse reaction) or, at the time of completing the study, have not completed two years of treatment. The effectiveness considered as the median number of cycles received at two years was 2 .

Conclusions Although the median time to retreatment was seven months, there are some patients who remain unplastered for about two years. $80 \%$ of the patients had been portrayed at 16 months, half with 1 cycle of RTX and the other half with 2 . The pattern on demand even allowed to decrease the doses of concomitant DMARDs. A subsequent individualised analysis of patients will allow us to evaluate the possible characteristics that can affect the effectiveness of the pattern on demand

Disclosure of interest None declared

\section{P123 THE IFN TYPE I GENE EXPRESSION IN A CARDIOVASCULAR DISEASE CONTINUUM OF RHEUMATOID ARTHRITIS}

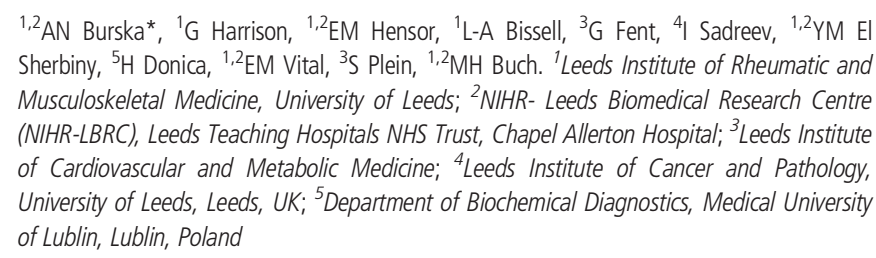

10.1136/annrheumdis-2018-EWRR2018.138

Introduction Rheumatoid Arthritis (RA) patients have a prominent increase in cardiovascular (CV) comorbidity not fully explained by traditional risk factors. ${ }^{1}$ Interferons type 1 (IFN1) have been associated with premature CV disease (CVD) in SLE $^{2}$ and are implicated in several aspects of atherosclerosis and acute coronary syndromes. ${ }^{3}$ In RA a subpopulation of patients also display an IFN-1 signature in the blood, ${ }^{4}$ which is associated with response to biologics. The IFN- 1 signature association with CVD in RA remains unclear.

Objectives To analyse expression of interferon type 1 response genes (ISGs) along a CVD continuum in patients with RA using a well phenotyped cohort of RA patients whereby 